\title{
Association of glycaemia with lipids in adults with type 1 diabetes: modification by dyslipidaemia medication
}

\author{
D. M. Maahs • L. G. Ogden • D. Dabelea • \\ J. K. Snell-Bergeon • S. R. Daniels • R. F. Hamman • \\ M. Rewers
}

Received: 12 May 2010 / Accepted: 19 July 2010 /Published online: 4 September 2010

(C) Springer-Verlag 2010

\begin{abstract}
Aims/hypothesis Hyperglycaemia and dyslipidaemia are common metabolic abnormalities in adults with type 1 diabetes and both increase cardiovascular disease (CVD) risk. The hypothesis of this study was that change in $\mathrm{HbA}_{1 \mathrm{c}}$ over 6 years would be associated with change in fasting lipids in adults with type 1 diabetes.

Methods The Coronary Artery Calcification in Type 1 Diabetes (CACTI) study examined 652 patients with type 1 diabetes (54\% female); 559 and 543 had follow-up visits at 3 and 6 years. Baseline age (mean \pm SD) was $37 \pm$ 9 years, diabetes duration $23 \pm 9$ years, and $\mathrm{HbA}_{1 \mathrm{c}} 8.0 \pm$ $1.3 \%$. Use of dyslipidaemia medication was $17 \%, 32 \%$, and $46 \%$ at the three visits. Separate longitudinal mixed models were fitted to examine the relationship between change in $\mathrm{HbA}_{1 \mathrm{c}}$ and change in fasting total cholesterol (TC),
\end{abstract}

D. M. Maahs · J. K. Snell-Bergeon · M. Rewers

Barbara Davis Center for Childhood Diabetes,

University of Colorado Denver,

Mail Stop A140, PO Box 6511, Aurora, CO 80045, USA

D. M. Maahs • D. Dabelea • S. R. Daniels • R. F. Hamman •

M. Rewers

Department of Epidemiology,

Colorado School of Public Health,

University of Colorado Denver,

Aurora, CO, USA

D. M. Maahs $(\bowtie) \cdot$ S. R. Daniels

The Children's Hospital Denver,

Aurora, CO, USA

e-mail: David.Maahs@ucdenver.edu

L. G. Ogden

Colorado School of Public Health,

Department of Biostatistics and Informatics,

University of Colorado Denver,

Aurora, CO, USA
HDL-cholesterol (HDL-c), LDL-cholesterol (LDL-c), log triacylglycerols (TG), and non-HDL-cholesterol (nonHDL-c). Because of an interaction between dyslipidaemia medication use and association of $\mathrm{HbA}_{1 \mathrm{c}}$ with lipids, results were stratified by dyslipidaemia medication use.

Results Among patients not using dyslipidaemia medication, a higher $\mathrm{HbA}_{1 \mathrm{c}}$ was associated with significantly worse levels of the lipids TC, LDL-c, TG and non-HDL-c (per $1 \%$ change in $\mathrm{HbAlc}$, TC $0.101 \mathrm{mmol} / 1,95 \% \mathrm{CI}$ 0.050, 0.152; LDL-c $0.103 \mathrm{mmol} / 1,95 \%$ CI $0.058,0.148$; TG $0.052 \mathrm{mmol} / 1,95 \%$ CI $0.024,0.081$; and non-HDL-c $0.129 \mathrm{mmol} / \mathrm{l}, 95 \%$ CI $0.078,0.180$ ) but not HDL-c $(-0.20 \mathrm{mmol} / 1,95 \% \mathrm{CI}-0.047,0.007)$. The associations between $\mathrm{HbA}_{1 \mathrm{c}}$ and any lipid outcome among those on dyslipidaemia medication were in the same direction, but attenuated compared with persons not on medication.

Conclusions/interpretation Change in $\mathrm{HbA}_{1 \mathrm{c}}$ is significantly associated with change in fasting lipids, but dyslipidaemia medications may be required to optimise lipid and cardiovascular health.

Keywords CVD risk - Dyslipidaemia medications . Glycaemia $\cdot$ Lipids $\cdot$ Type 1 diabetes

$\begin{array}{ll}\text { Abbreviations } \\ \text { CACTI } & \text { Coronary Artery Calcification in Type 1 Diabetes } \\ \text { CAD } & \text { Coronary artery disease } \\ \text { CVD } & \text { Cardiovascular disease } \\ \text { DCCT } & \text { Diabetes Control and Complications Trial } \\ \text { EDC } & \text { Epidemiology of Diabetes Complications } \\ \text { EDIC } & \text { Epidemiology of Diabetes Control } \\ \text { HDL-c } & \text { HDL-cholesterol } \\ \text { LDL-c } & \text { LDL-cholesterol } \\ \text { TC } & \text { Total cholesterol } \\ \text { TG } & \text { Triacylglycerols }\end{array}$




\section{Introduction}

Hyperglycaemia, as measured by $\mathrm{HbA}_{1 \mathrm{c}}$, is the most important determinant of vascular complications of type 1 diabetes [1-4]. In addition to hyperglycaemia, dyslipidaemia is a well-established cardiovascular disease (CVD) risk factor [5, 6]. However, few data exist in people with type 1 diabetes on the relationship of glycaemia to other CVD risk factors such as dyslipidaemia, although aggressive control of glycaemia is recommended as first line treatment of dyslipidaemia in the care of people with type 1 diabetes, with addition of lipid-lowering medications if lipid goals are not achieved [7, 8]. Although hyperglycaemia and dyslipidaemia [9] both increase CVD risk and are metabolic abnormalities frequently found in individuals with type 1 diabetes, it is unclear how changes in $\mathrm{HbA}_{1 \mathrm{c}}$ are associated with alterations in lipids and whether dyslipidaemia medications modify this association.

The Diabetes Control and Complications Trial (DCCT) demonstrated that intensive (vs conventional) insulin therapy was associated with significantly reduced total cholesterol (TC), LDL-cholesterol (LDL-c) and triacylglycerols (TG) $(p<$ 0.01 for all) and decreased development of LDL-c $>4.14 \mathrm{mmol} / \mathrm{l}$ in adults with type 1 diabetes, which was considered a treatment threshold at the time of the study [10], as well as a more favourable distribution of lipoprotein cholesterol [11]. Similarly, the DCCT/Epidemiology of Diabetes Control (EDIC) study demonstrated that intensive vs conventional insulin therapy was associated with a $24 \%$ reduced risk of developing incident hypertension over a median of 15.8 years of follow-up [12]. Thus, it is plausible that some of the deleterious effect of hyperglycaemia on cardiovascular health is mediated via the effects of hyperglycaemia on hypertension and dyslipidaemia $[10,12]$.

Therefore, to better describe the relationship of glycaemia to dyslipidaemia outside the setting of a clinical trial, we tested the hypothesis that change in $\mathrm{HbA}_{1 \mathrm{c}}$ over a follow-up interval of approximately 6 years resulted in a concordant change in the lipid profile in adults with type 1 diabetes participating in the Coronary Artery Calcification in Type 1 Diabetes (CACTI) study, independent of potential confounders, and accounting for dyslipidaemia medication usage.

\section{Methods}

Study participants The data presented in this report were collected as part of the baseline examination of 1,416 participants in the CACTI study who were 19-56 years of age and included 652 participants with type 1 diabetes [13]. All participants were asymptomatic for coronary artery disease (CAD) and had no history of coronary artery bypass graft, coronary angioplasty, or unstable angina. Participants with diabetes generally had been diagnosed when $<30$ years of age, or had positive antibodies or a clinical course consistent with type 1 diabetes. Of the 652 persons enrolled at baseline, $559(86 \%)$ participated in a second study visit $\sim 3$ years after baseline and $543(83 \%)$ in a third study visit $\sim 6$ years after baseline, with $603(92 \%)$ of participants having at least one follow-up study visit. Participants who did not have any follow-up data $(n=49)$ had higher TC, LDL-c and non-HDL-cholesterol (non-HDL-c) at baseline than those with follow-up data. All participants provided informed consent and the study was approved by the Colorado Multiple Institutional Review Board.

Examination and laboratory measurements Participants completed a baseline examination between March 2000 and April 2002. A more detailed description of the study and baseline characteristics of this cohort has been published [14]. The second and third study visits followed the same protocol and occurred between 2003 and 2005 and between 2006 and 2008, respectively.

Laboratory analyses Blood was collected after an overnight fast and serum was stored at $-70^{\circ} \mathrm{C}$ until assayed. Fasting plasma samples were obtained for measurement of TC, HDL-c and TG, in the University of Colorado Denver Clinical Translational Research Center core lab. Measurements of TC, HDL-c, and TG were performed enzymatically on a Hitachi 917 autoanalyser (Boehringer Mannheim Diagnostics, Indianapolis, IN, USA). LDL-c levels were calculated by the Friedewald equation for individuals with TG levels lower than $4.52 \mathrm{mmol} / 1$ [15] and by Lipid Research Clinics Beta Quantification [16] for those with $\mathrm{TG} \geq 4.52 \mathrm{mmol} / \mathrm{l}$. Non-HDL-c was calculated as TC minus HDL-c. HbA $_{1 \mathrm{c}}$ levels were measured by (DCCT-calibrated) ion-exchange high-performance liquid chromatography (Bio-Rad Laboratories, Hercules, CA, USA).

Variables Weight and height were measured using standard methods and BMI, defined as weight $(\mathrm{kg})$ divided by height $\left(\mathrm{m}^{2}\right)$, was calculated. Minimal waist circumference (measured at the smallest point between the tenth rib and the iliac crest over the bare skin) was measured. Intraabdominal fat and subcutaneous fat were assessed using abdominal computed tomography scan at the L4-L5 levels and volume in cubic centimeters was measured using the AccuAnalyzer software from AccuImage.

Dyslipidaemia medications Participants brought all current medications to each study visit for documentation by study staff. Dyslipidaemia treatment was defined as current use of lipid-lowering medication, such as hydroxymethylglutarylCoA reductase inhibitors, bile acid sequestrants, fibric acid derivatives, ezetimibe or niacin, and was recorded at each 
study visit. Dyslipidaemia medication treatment decisions were made by the participants' physicians, not by the CACTI study team.

Statistical methods The mean and SE for each variable of interest at baseline and follow-up were estimated using the SAS Mixed procedure for linear mixed models. TG was log transformed due to non-normal distribution. Therefore, back transformed estimates were calculated as change in TG per $1 \%$ change in $\mathrm{HbA}_{1 \mathrm{c}}$ from a starting level of $0.93 \mathrm{mmol} / \mathrm{l}$ (median baseline TG level for cohort.) To determine the association of change in $\mathrm{HbA}_{1 \mathrm{c}}$ with change in lipids, two a priori models (basic and saturated) were constructed with separate models for change in each lipid variable (TC, HDL-c, TG, LDL-c, and non-HDL-c) as an outcome. Time-varying change variables were created for $\mathrm{HbA}_{1 \mathrm{c}}$ and each lipid outcome for change from baseline to the first follow-up visit and from baseline to the second follow-up visit. The outcome of interest in all models was change in lipid levels over time. Baseline lipid levels were also included as a covariate to increase precision. In the basic model, demographic variables (sex, race/ethnicity, diabetes duration, and age as a time-varying variable) were adjusted for in addition to change in $\mathrm{HbA}_{1 \mathrm{c}}$, visit, and a change in $\mathrm{HbA}_{1 \mathrm{c}}$ by visit interaction term to determine whether the association of change in $\mathrm{HbA}_{1 \mathrm{c}}$ with change in lipid outcome differed by visit. To account for differences in baseline levels of $\mathrm{HbA}_{1 \mathrm{c}}$, baseline $\mathrm{HbA}_{1 \mathrm{c}}$ was also included. A baseline $\mathrm{HbA}_{1 \mathrm{c}}$ by time-varying change in $\mathrm{HbA}_{1 \mathrm{c}}$ interaction term was tested for to determine whether the association of change in $\mathrm{HbA}_{1 \mathrm{c}}$ with change in lipid was different according to baseline $\mathrm{HbA}_{1 \mathrm{c}}$, but this was not significant and was thus not included in the final models. Since the change in $\mathrm{HbA}_{1 \mathrm{c}}$ by visit interaction terms were not significant, ESTIMATE statements were used to determine the average association of change in $\mathrm{HbA}_{1 \mathrm{c}}$ with change in each lipid outcome over time. In the saturated model, to account for the confounding role of adiposity on lipids, we additionally adjusted for BMI, waist circumference and visceral fat as time-varying variables. All three of these measures were included to fully capture the confounding effect of adiposity on lipids, as the individual role of each adiposity measure is not the focus of this analysis. Dyslipidaemia medication usage was considered as an effect modifier for the association of $\mathrm{HbA}_{1 \mathrm{c}}$ with lipid levels. A time-varying dyslipidaemia medication usage (yes/no) by change in $\mathrm{HbA}_{1 \mathrm{c}}$ interaction term was included to determine whether the association of change in $\mathrm{HbA}_{1 \mathrm{c}}$ with change in the lipid outcome differed according to whether or not a participant was on a dyslipidaemia medication. As significant differences existed, models stratified by dyslipidaemia medication usage status are presented. In post hoc analyses, interactions for both time- varying age and sex by change in $\mathrm{HbA}_{1 \mathrm{c}}$ were explored and, since significant differences existed by sex, models stratified by sex are also presented. The Mixed procedure in SAS 9.2 (SAS Institute, Cary, NC, USA) was used for the analysis. A $p$ value of $<0.05$ was considered statistically significant.

\section{Results}

Statistically significant improvements over 6 years (Table 1 , $p<0.0001$ for all) were noted for TC (mean decrease of $0.26 \mathrm{mmol} / \mathrm{l}$ ), HDL-c (mean increase of $0.13 \mathrm{mmol} / \mathrm{l}$ ), LDL-c (mean decrease of $0.33 \mathrm{mmol} / \mathrm{l})$, TG (mean decrease of $0.14 \mathrm{mmol} / \mathrm{l}$ ) and non-HDL-c (mean decrease of $0.39 \mathrm{mmol} / \mathrm{l}$ ). $\mathrm{HbA}_{1 \mathrm{c}}$ also improved over time (mean decrease of $0.24 \%$ from baseline to first follow-up and mean decrease of $0.08 \%$ from baseline to second followup). Reported use of dyslipidaemia medications increased from $17 \%$ at baseline to $32 \%$ at the first follow-up and to $46 \%$ at the second follow-up visit. The improvements in lipid measures were noted despite increases in adiposity measures over the 6 year period.

The general pattern for reported dyslipidaemia medication use was that once participants started medications they continued on medications. Between the baseline and first follow-up visit, 85 additional participants were started on dyslipidaemia medications while 13 participants reported discontinuing dyslipidaemia medications. From the baseline to second follow-up visit 79 participants began and 13 discontinued dyslipidaemia medications. Of those on dyslipidaemia medications $97 \%, 95 \%$ and $93 \%$ were on statins at each visit.

In non-stratified models, there was a significantly different association of change in $\mathrm{HbA}_{1 \mathrm{c}}$ with change in lipid outcomes (TC, LDL-c, and non-HDL-c, $p<0.0001$ for each $\mathrm{HbA}_{1 \mathrm{c}}$ by dyslipidaemia medication use interaction term) depending on whether a participant reported use of dyslipidaemia medication. We therefore report the results of stratified mixed model analysis of the association of change in $\mathrm{HbA}_{1 \mathrm{c}}$ to change in each lipid outcome ( $\beta$-estimate $\pm \mathrm{SE}$ and $p$ value) in the basic and saturated models as shown in Table 2. Among those not on dyslipidaemia medications, statistically significant associations existed for the association between change in HbAlc and change in TC, LDL-c, TG, and non-HDL-c, but not HDL-c. In the basic model, for every $1 \%$ change in $\mathrm{HbA}_{1 \mathrm{c}}$ the associated changes in lipids over 6 years were: $\mathrm{TC}+0.101 \mathrm{mmol} / 1 \mathrm{(95 \%}$ CI 0.050 , $0.152, p=0.0001)$, HDL-c $-0.20 \mathrm{mmol} / 1(95 \% \mathrm{CI}-0.047$, $0.007, p=0.15)$, LDL-c $+0.103 \mathrm{mmol} / 1 \quad(95 \%$ CI 0.058 , $0.148, p<0.0001)$, TG $+0.052 \mathrm{mmol} / 1 \quad(95 \%$ CI 0.024 , $0.081, p=0.0003)$, and non-HDL-c $+0.129 \mathrm{mmol} / 1$ (95\% CI $0.078,0.180, p<0.0001)$. Similar associations existed in the 
Table 1 Characteristics of CACTI Type 1 Diabetes Cohort

\begin{tabular}{|c|c|c|c|c|}
\hline Variable & Visit $0, n=652$ & Visit $1, n=559$ & Visit $2, n=543$ & $p$ value \\
\hline Age (years) & $36.5 \pm 0.4$ & $39.0 \pm 0.4$ & $43.2 \pm 0.4$ & NA \\
\hline Type 1 diabetes duration (years) & $23.3 \pm 0.3$ & $25.8 \pm 0.3$ & $29.6 \pm 0.3$ & NA \\
\hline Time to follow-up (years) & NA & $2.5 \pm 0.4$ & $6.2 \pm 0.6$ & NA \\
\hline Sex (male/female), $\%$ & $46 \% / 54 \%$ & $46 \% / 54 \%$ & $46 \% / 54 \%$ & NA \\
\hline Race/ethnicity (NHW), \% & $94 \%$ & $94 \%$ & $94 \%$ & NA \\
\hline $\mathrm{HbA}_{1 \mathrm{c}}(\%)$ & $7.98 \pm 0.05$ & $7.74 \pm 0.05$ & $7.90 \pm 0.05$ & $<0.0001$ \\
\hline $\mathrm{TC}(\mathrm{mmol} / \mathrm{l})$ & $4.53 \pm 0.03$ & $4.60 \pm 0.04$ & $4.27 \pm 0.04$ & $<0.0001$ \\
\hline HDL (mmol/l) & $1.45 \pm 0.02$ & $1.58 \pm 0.02$ & $1.58 \pm 0.02$ & $<0.0001$ \\
\hline LDL (mmol/l) & $2.61 \pm 0.03$ & $2.61 \pm 0.03$ & $2.28 \pm 0.03$ & $<0.0001$ \\
\hline $\mathrm{TG}^{\mathrm{a}}(\mathrm{mmol} / \mathrm{l})$ & $0.93(0.28-7.54)$ & $0.75(0.30-6.58)$ & $0.79(0.28-3.92)$ & $<0.0001$ \\
\hline Non-HDL (mg/l) & $3.08 \pm 0.03$ & $3.00 \pm 0.03$ & $2.69 \pm 0.04$ & $<0.0001$ \\
\hline Dyslipidaemia medications, yes/total, $\%$ & $113 / 652(17 \%)$ & $180 / 559(32 \%)$ & $250 / 543(46 \%)$ & $<0.0001$ \\
\hline BMI $\left(\mathrm{kg} / \mathrm{m}^{2}\right)$ & $26.2 \pm 0.2$ & $26.5 \pm 0.2$ & $26.9 \pm 0.2$ & $<0.0001$ \\
\hline Waist circumference $(\mathrm{cm})$ & $85.1 \pm 0.5$ & $85.2 \pm 0.5$ & $88.7 \pm 0.5$ & $<0.0001$ \\
\hline $\operatorname{VSF}\left(\mathrm{cm}^{3}\right)$ & $10.3 \pm 0.03$ & $10.5 \pm 0.03$ & $10.6 \pm 0.02$ & $<0.0001$ \\
\hline
\end{tabular}

Results are presented as means $\pm \mathrm{SE}$ or count and frequency, except for 'Time to follow-up' (mean $\pm \mathrm{SD}$ )

$p$ value, type 3 tests of fixed effects by visit in SAS Proc Mixed

$n=603$ with a baseline and either first or second visit

${ }^{\mathrm{a}}$ Geometric mean and range

NA, not applicable; NHW, non-Hispanic white; TC, total cholesterol; TG, triacylglycerols; VSF, visceral fat

saturated model (Table 2). In contrast, among those on dyslipidaemia medications, the association of change in $\mathrm{HbA}_{1 \mathrm{c}}$ with change in lipid level was reduced by approximately one-half and was no longer statistically significant, though the direction of the effect was similar to persons not on medication. Figure 1 displays the association of change in $\mathrm{HbA}_{1 \mathrm{c}}$ and change in lipid.

In participants not on dyslipidaemia medications, the following variables were also significantly associated with lipid outcomes in the basic model: male sex (HDL-c, LDL-c, non-HDL-c), older age (TC, HDL-c, LDL-c and nonHDL-c) and in the saturated models larger waist (HDL-c) and higher visceral fat (HDL-c, TG and non-HDL-c). Among participants on dyslipidaemia medications the following variables were significantly associated with lipid outcomes in the basic model: race/ethnicity other than nonHispanic white (TG), higher baseline $\mathrm{HbA}_{1 \mathrm{c}}$ (all) and higher baseline lipid levels (all); and in the saturated models: male sex (HDL-c), older age (TC), higher baseline $\mathrm{HbA}_{1 \mathrm{c}}$ (all) and higher baseline lipid levels (all).

In post hoc analyses, significant differences existed in the association of $\mathrm{HbA}_{1 \mathrm{c}}$ with lipids, and models stratified by sex are presented in Tables 3 and 4. In men, there was a stronger association of $\mathrm{HbA}_{1 \mathrm{c}}$ with TC, LDL-c and non-HDL-c. $\mathrm{HbA}_{1 \mathrm{c}}$ was not associated with HDL-c in either sex. In men, $\mathrm{HbA}_{1 \mathrm{c}}$ was associated with TG in those not on dyslipidaemia medications in the basic but not the saturated model, and was of borderline significance in those on dyslipidaemia medications, whereas in women $\mathrm{HbA}_{1 \mathrm{c}}$ was significantly associated with TG in those not on dyslipidaemia medications, but not in those on dyslipidaemia medications.

\section{Discussion}

We found that over 6 years of follow-up an increase in glycaemia was positively associated with change in TC, LDL-c, TG and non-HDL-c in adults with type 1 diabetes who were not on dyslipidaemia medications. This strong association was not observed in patients who were on dyslipidaemia medications. Although glycaemic control is important for reduction of vascular complications, we believe these data suggest that the effect of dyslipidaemia treatment on lipids is stronger than the effect of glycaemia on lipids and, therefore, may obscure the effect of glucose control on lipids. Therefore, this suggests that both glucose control and treatment with dyslipidaemia medications (if lipids exceed treatment goals) in adults with type 1 diabetes should be emphasised to achieve lipid and cardiovascular health. Furthermore, given the magnitude of the association of change in $\mathrm{HbA}_{1 \mathrm{c}}$ with change in lipids, for some patients with significantly elevated lipids it is likely that intensification of glycaemic control may be insufficient to achieve lipid treatment goals $[7,17]$. 
Table 2 Longitudinal change in lipid per $1 \%$ point increase in $\mathrm{HbA}_{1 \mathrm{c}}$ estimate

\begin{tabular}{lllll}
\hline & $\begin{array}{l}\text { Basic model } \\
\beta(95 \% \mathrm{CI})\end{array}$ & $p$ value & $\begin{array}{l}\text { Saturated model } \\
\beta(95 \% \mathrm{CI})\end{array}$ \\
\hline $\begin{array}{l}\text { NO lipid medications } \\
\text { TC }\end{array}$ & $0.101(0.050,0.152)$ & & & \\
HDL-c & $-0.020(-0.047,0.007)$ & 0.0001 & $0.127(0.072,0.182)$ & $<0.0001$ \\
LDL-c & $0.103(0.058,0.148)$ & 0.15 & $-0.008(-0.035,0.019)$ & 0.57 \\
TG & $0.052(0.024,0.081)$ & $<0.0001$ & $0.1220 .071,0.173)$ & $0.043(0.014,0.073)$ \\
Non-HDL-c & $0.129(0.078,0.180)$ & 0.0003 & $0.140(0.085,0.195)$ & 0.0004 \\
YES lipid medications & & $<0.0001$ & $0.036(-0.055,0.127)$ \\
TC & $0.052(-0.034,0.138)$ & & $-0.022(-0.059,0.015)$ \\
HDL-c & $-0.018(-0.053,0.017)$ & 0.23 & $0.054(-0.26,0.134)$ \\
LDL-c & $0.062(-0.014,0.138)$ & 0.32 & $0.027(-0.016,0.072)$ \\
TG & $0.025(-0.018,0.070)$ & 0.12 & $0.065(-0.021,0.151)$ \\
Non-HDL-c & $0.072(-0.008,0.152)$ & 0.25 & 0.0001 \\
\hline
\end{tabular}

Basic model includes sex, visit, race/ethnicity, diabetes duration, baseline $\mathrm{HbA}_{1 \mathrm{c}}$ and baseline lipid (model-specific) and time-varying age, change in $\mathrm{HbA}_{1 \mathrm{c}}$, change in $\mathrm{HbA}_{1 \mathrm{c}} \times$ visit interaction

Saturated model includes variables included in the basic model plus time-varying BMI, waist circumference and visceral fat

${ }^{\text {a }}$ Change in TG per $1 \%$ change in $\mathrm{HbA}_{\mathrm{lc}}$ from a starting level of $0.93 \mathrm{mmol} / \mathrm{l}$

In post hoc analyses, we found that the association of $\mathrm{HbA}_{1 \mathrm{c}}$ with TC, LDL-c and non-HDL-c was of reduced magnitude in women as compared with men. $\mathrm{HbA}_{1 \mathrm{c}}$ was not associated with HDL-c in either men or women, and the association of $\mathrm{HbA}_{1 \mathrm{c}}$ with $\mathrm{TG}$ was significant only in

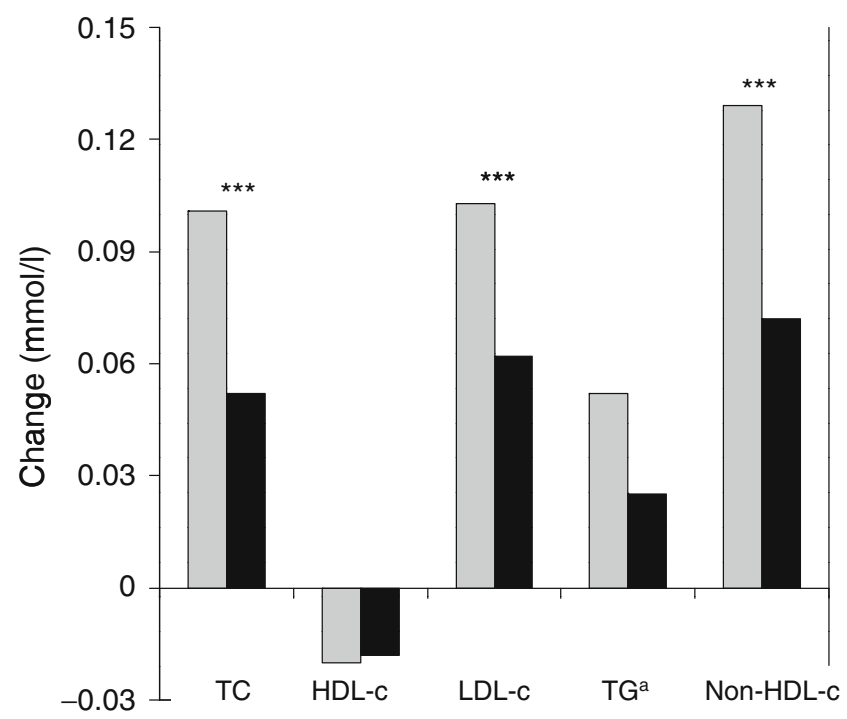

Fig. 1 Longitudinal association of change in lipid per 1\% change in $\mathrm{HbA}_{1 \mathrm{c}}$ for the basic model (adjusted for sex, visit, race/ethnicity, diabetes duration, baseline $\mathrm{HbA}_{1 \mathrm{c}}$ and baseline lipid [model-specific] and time-varying age, change in $\mathrm{HbA}_{1 \mathrm{c}}$, and change in $\mathrm{HbA}_{1 \mathrm{c}} \times$ visit interaction). Grey bars, participants receiving no lipid medication; black bars, participants receiving lipid medication. ${ }^{* * * *} p<0.001$ for dyslipidaemia medication status for change per $1 \%$ increase in $\mathrm{HbA}_{1 \mathrm{c}}$. ${ }^{a}$ Estimated change in TG from a starting level of $0.93 \mathrm{mmol} / \mathrm{l}$ women not on dyslipidaemia medications, whereas men had a more variable pattern. Sex-based differences in CVD risk in type 1 diabetes have been previously reported [18-20] and we have reported that insulin resistance was associated with a proatherogenic lipoprotein subfraction cholesterol distribution in women [21]. Further investigation of sexbased differences in CVD risk in type 1 diabetes is needed.

CACTI is an observational cohort, and use of dyslipidaemia medications increased from $17 \%$ to $46 \%$ over the mean 6 years of follow-up, demonstrating increased use of dyslipidaemia medications for lipid and cardiovascular health in type 1 diabetes. Not surprisingly, the fasting lipid profile improved over the study period despite increases in adiposity measures. In contrast, there was minimal average improvement in $\mathrm{HbA}_{1 \mathrm{c}}$ over the study period. In the DCCT trial, TC, LDL-c and TG were significantly lower in the intensively treated arm than in the conventionally treated arm [10]. In addition, no participants were on statins at the end of the DCCT in 1993, while in 2004 in EDIC 33\% and $34 \%$ in the intensive and conventional arms, respectively, were on statins [22]. The improved lipid profile in CACTI over time is likely due to increased use of dyslipidaemia medications, not improved glycaemic control.

Data clearly suggest that care for type 1 diabetes has improved in the past two decades, as evidenced by reduced rates of microvascular disease [23-25]; however, substantially less progress has been made in reduction of macrovascular disease rates [25-27], despite the DCCT/EDIC study showing that intensive glycaemic control over a mean of 6.5 years reduced CVD complications by $57 \%$ after a 


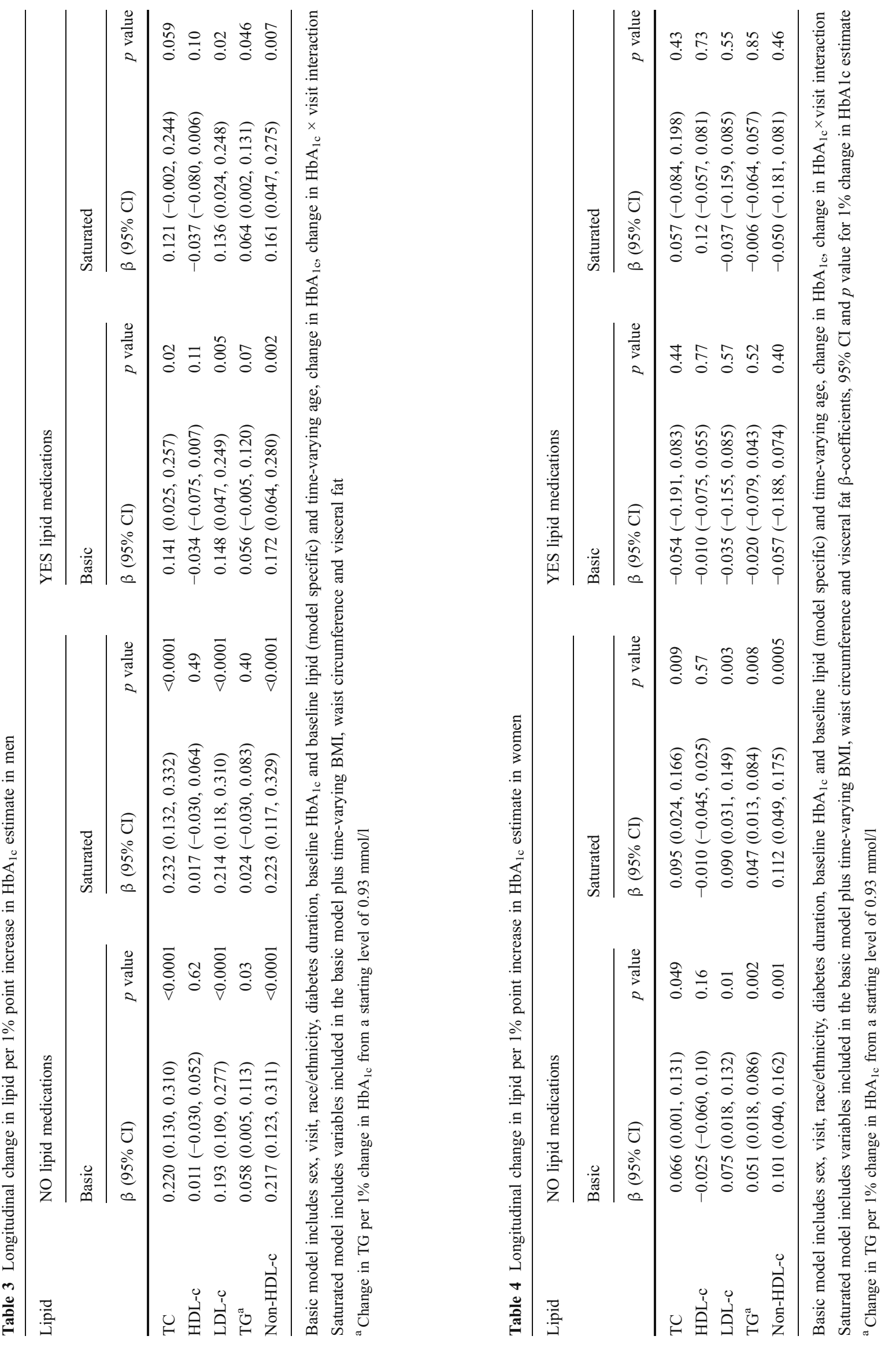


mean of 17 years of follow-up [22]. People with type 1 diabetes continue to have excess morbidity and mortality, with a dramatic increase in CVD mortality risk compared with people without diabetes [27].

To describe the clinical course of type 1 diabetes in the post-DCCT age of intensive insulin therapy, data from 161 participants of the Pittsburgh Epidemiology of Diabetes Complications (EDC) Study ( $n=88$ at the 2006 follow-up) were compared with those from the DCCT/EDIC Study [28]. The EDC reported a mean $\mathrm{HbA}_{1 \mathrm{c}}$ similar to that of the conventional arm of the DCCT (9\%) until EDC year 8 (1994-1996), when $\mathrm{HbA}_{1 \mathrm{c}}$ fell by $0.5 \%$, subsequent to publication of initial data from the DCCT demonstrating reduced microvascular complications with intensive control [28]. Subjects in the observational EDC reported a decrease in $\mathrm{HbA}_{1 \mathrm{c}}$ from $9.0 \%$ to $8.3 \%$, an increase in BMI from 24 to $28 \mathrm{~kg} / \mathrm{m}^{2}$, and an increase in statin use from $1 \%$ to $38 \%$ from 1986-1988 to 2006, similar to the CACTI data. The authors emphasise that, based on these findings, current outcomes for people with type 1 diabetes should be much improved in comparison to cohorts who had onset of type 1 diabetes in the 1950s to 1970 s.

In addition to glycaemic control, dyslipidaemia is another important CVD risk factor with extensive data to support its role as a target to improve cardiovascular health in people with type 1 diabetes [5]. Despite abundant data on the importance of controlling dyslipidaemia, adequate control of this CVD risk factor is frequently not achieved [9]. Our results add to those of the DCCT/EDIC and EDC studies by providing data on glycaemic and lipid control in a large observational cohort in the early 21 st century. Moreover, we investigate the interplay of glycaemic control and dyslipidaemia medication use on the fasting lipid profile over a 6 year period in a large cohort of adults with type 1 diabetes. As the DCCT/EDIC study has shown that hyperglycaemia is a risk factor for hypertension [12], our data support a similar role for hyperglycaemia as a risk factor for a more atherogenic lipid profile; however, the association of glycaemia with lipids is attenuated by dyslipidaemia medication use in CACTI, whereas in the DCCT/EDIC study the association of intensive therapy with reduced incidence of hypertension remained significant in sensitivity analyses when accounting for use of antihypertensive medications [12].

While intensive treatment of glycaemia has been shown to reduce vascular complications in type 1 diabetes [22], there is also concern that intensive control is associated with weight gain, leading to adverse effects on blood pressure and lipids [29]. In the CACTI cohort, $\mathrm{HbA}_{1 \mathrm{c}}$ was relatively stable, adiposity measures increased and lipids improved over time. How this improved lipid profile translates into changes in health outcomes remains an important future research objective. Limited clinical trial data exist on the effect of dyslipidaemia medications on lipids and outcomes in type 1 diabetes, but the Heart Protection Study included 615 participants with type 1 diabetes. Though the study was underpowered to detect an effect in subgroup analysis, the magnitude of the risk reduction was similar to that in type 2 diabetes [30], consistent with a subsequent meta-analysis [31].

As noted, CACTI is an observational cohort study and not the result of a randomised clinical trial; therefore our data have limitations to consider when applying these findings to clinical scenarios. While $8 \%$ of participants did not have at least one follow-up visit, minimal differences exist between those who did and did not follow up. We also did not measure the effect of change in diet and exercise on change in lipid levels in these analyses, although these effects would likely be captured in the adiposity measures, which were adjusted for in the analyses. Nonetheless, CACTI participants represent one of the largest cohorts of persons with type 1 diabetes followed over 6 years who have had detailed and repeated measures of glycaemia, lipids, dyslipidaemia medications and adiposity. Our analyses stratified by sex were post hoc and should be further investigated. Finally, sensitivity analyses were performed in which participants with serum creatinine $>2.0 \mathrm{mg} / \mathrm{dl}$ or microalbuminuria were removed from the analysis, and the associations of change in $\mathrm{HbA}_{1 \mathrm{c}}$ with change in lipids were similar.

In conclusion, over a 6 year period in the CACTI cohort, change in glycaemia was associated with a concomitant change in TC, LDL-c, TG and non-HDL-c, which was not observed in the participants who were on dyslipidaemia medications. These data suggest that there is a statistically strong association between change in glycaemia and change in lipid levels; however, this association may not be as clinically relevant compared with the stronger effect of treatment with dyslipidaemia medications on lipid levels. Therefore, both glycaemic control and treatment with dyslipidaemia medications, when indicated, in adults with type 1 diabetes may be required to optimise lipid and cardiovascular health and improve patient outcomes. Further data are needed to inform clinical decisions on lipid lowering in type 1 diabetes.

Acknowledgements Support for this study was provided by the National Institutes of Health National Heart Lung Blood Institute grant R01 HL61753, HL79611, and Diabetes Endocrinology Research Center Clinical Investigation Core P30 DK57516. The study was performed at the Adult General Clinical Research Center at University of Colorado Denver supported by grant no. NIH-M01-RR00051, at the Barbara Davis Center for Childhood Diabetes and at Colorado Heart Imaging Center in Denver (CO, USA). D. M. Maahs was supported by grant no. K23 DK075360. The results presented in this paper have not been published previously in whole or part, except in abstract format (American Diabetes Association Scientfic Sessions, Orlando, FL, USA, 2010). 
Duality of interest D. M. Maahs has research support from Merck for a separate trial of dyslipidaemia medications in adolescents with type 1 diabetes. None of the other authors has a duality of interest associated with this manuscript.

\section{References}

1. Nathan DM, Cleary PA, Backlund JY et al (2005) Intensive diabetes treatment and cardiovascular disease in patients with type 1 diabetes. N Engl J Med 353:2643-2653

2. Snell-Bergeon JK, Hokanson JE, Jensen L et al (2003) Progression of coronary artery calcification in type 1 diabetes: the importance of glycemic control. Diab Care 26:2923-2928

3. The Diabetes Control and Complications Trial Research Group (1993) The effect of intensive treatment of diabetes on the development and progression of long-term complications in insulin-dependent diabetes mellitus. N Engl J Med 329:977-986

4. Diabetes Control and Complications Trial Research Group (1994) Effect of intensive diabetes treatment on the development and progression of long-term complications in adolescents with insulin-dependent diabetes mellitus: Diabetes Control and Complications Trial. J Pediatr 125:177-188

5. American Diabetes Association (2008) Standards of medical care in diabetes - 2008. Diab Care 31(Suppl 1):S12-S54

6. Maahs DM, Wadwa RP, Bishop F, Daniels SR, Rewers M, Klingensmith GJ (2008) Dyslipidemia in youth with diabetes: to treat or not to treat? J Pediatr 153:458-465

7. Buse JB, Ginsberg HN, Bakris GL et al (2007) Primary prevention of cardiovascular diseases in people with diabetes mellitus: a scientific statement from the American Heart Association and the American Diabetes Association. Diab Care 30:162-172

8. Brunzell JD, Davidson M, Furberg CD et al (2008) Lipoprotein management in patients with cardiometabolic risk: consensus statement from the American Diabetes Association and the American College of Cardiology Foundation. Diab Care 31:811-822

9. Wadwa RP, Kinney GL, Maahs DM et al (2005) Awareness and treatment of dyslipidemia in young adults with type 1 diabetes. Diab Care 28:1051-1056

10. The Diabetes Control and Complications Trial (DCCT) Research Group (1995) Effect of intensive diabetes management on macrovascular events and risk factors in the Diabetes Control and Complications Trial. Am J Cardiol 75:894-903

11. Purnell JQ, Marcovina SM, Hokanson JE et al (1995) Levels of lipoprotein(a), apolipoprotein B, and lipoprotein cholesterol distribution in IDDM. Results from follow-up in the Diabetes Control and Complications Trial. Diabetes 44:1218-1226

12. de Boer IH, Kestenbaum B, Rue TC et al (2008) Insulin therapy, hyperglycemia, and hypertension in type 1 diabetes mellitus. Arch Intern Med 168:1867-1873

13. Maahs DM, Kinney GL, Wadwa P et al (2005) Hypertension prevalence, awareness, treatment, and control in an adult type 1 diabetes population and a comparable general population. Diab Care 28:301-306

14. Dabelea D, Kinney G, Snell-Bergeon JK et al (2003) Effect of type 1 diabetes on the gender difference in coronary artery calcification: a role for insulin resistance? The Coronary Artery Calcification in Type 1 Diabetes (CACTI) Study. Diabetes 52:2833-2839
15. Friedewald WT, Levy RI, Fredrickson DS (1972) Estimation of the concentration of low-density lipoprotein cholesterol in plasma, without use of the preparative ultracentrifuge. Clin Chem 18:499-503

16. Hainline A Jr, Miller DT, Mather A (1983) The Coronary Drug Project. Role and methods of the Central Laboratory. Control Clin Trials 4:377-387

17. De Backer G, Ambrosioni E, Borch-Johnsen K et al (2003) European guidelines on cardiovascular disease prevention in clinical practice. Third Joint Task Force of European and Other Societies on Cardiovascular Disease Prevention in Clinical Practice. Eur Heart J 24:1601-1610

18. Lloyd CE, Kuller LH, Ellis D, Becker DJ, Wing RR, Orchard TJ (1996) Coronary artery disease in IDDM. Gender differences in risk factors but not risk. Arterioscler Thromb Vasc Biol 16:720-726

19. Colhoun HM, Rubens MB, Underwood SR, Fuller JH (2000) The effect of type 1 diabetes mellitus on the gender difference in coronary artery calcification. J Am Coll Cardiol 36:2160-2167

20. Laing SP, Swerdlow AJ, Slater SD et al (2003) Mortality from heart disease in a cohort of 23,000 patients with insulin-treated diabetes. Diabetologia 46:760-765

21. Maahs DM, Hokanson JE, Wang H et al (2010) Lipoprotein subfraction cholesterol distribution is proatherogenic in women with type 1 diabetes and insulin resistance. Diabetes 59:1771-1779

22. Nathan DM, Cleary PA, Backlund JY et al (2005) Intensive diabetes treatment and cardiovascular disease in patients with type 1 diabetes. N Engl J Med 353:2643-2653

23. Maahs DM, Rewers M (2006) Editorial: Mortality and renal disease in type 1 diabetes mellitus - progress made, more to be done. J Clin Endocrinol Metab 91:3757-3759

24. Hovind P, Tarnow L, Rossing K et al (2003) Decreasing incidence of severe diabetic microangiopathy in type 1 diabetes. Diab Care 26:1258-1264

25. Pambianco G, Costacou T, Ellis D, Becker DJ, Klein R, Orchard TJ (2006) The 30-year natural history of type 1 diabetes complications: the Pittsburgh Epidemiology of Diabetes Complications Study experience. Diabetes 55:1463-1469

26. Libby P, Nathan DM, Abraham K et al (2005) Report of the National Heart, Lung, and Blood Institute-National Institute of Diabetes and Digestive and Kidney Diseases Working Group on Cardiovascular Complications of Type 1 Diabetes Mellitus. Circulation 111:3489-3493

27. Rewers M (2008) Why do people with diabetes die too soon? More questions than answers. Diab Care 31:830-832

28. Nathan DM, Zinman B, Cleary PA et al (2009) Modern-day clinical course of type 1 diabetes mellitus after 30 years' duration: the diabetes control and complications trial/epidemiology of diabetes interventions and complications and Pittsburgh epidemiology of diabetes complications experience (1983-2005). Arch Intern Med 169:1307-1316

29. Purnell JQ, Hokanson JE, Marcovina SM, Steffes MW, Cleary PA, Brunzell JD (1998) Effect of excessive weight gain with intensive therapy of type 1 diabetes on lipid levels and blood pressure: results from the DCCT. Diabetes Control and Complications Trial. JAMA 280:140-146

30. Collins R, Armitage J, Parish S, Sleigh P, Peto R (2003) MRC/BHF Heart Protection Study of cholesterol-lowering with simvastatin in 5,963 people with diabetes: a randomised placebo-controlled trial. Lancet 361:2005-2016

31. Kearney PM, Blackwell L, Collins R et al (2008) Efficacy of cholesterol-lowering therapy in 18,686 people with diabetes in 14 randomised trials of statins: a meta-analysis. Lancet 371:117-125 